\title{
Die fossilen Damhirsche von Neumark-Nord (Sachsen-Anhalt) - D. dama geiselana n. ssp.
}

\author{
TheKla Pfeiffer*) \\ Vertebrate Paleontology, Pleistocene, new Subspemics of Dama dama (Cervidae, \\ Mammalia), fossil follow deer population
}

\begin{abstract}
Kurzfassung: Die Fundstelle Neumark-Nord am Nordrand des Geiseltals (SW von Halle, Sachsen-Anhalt, Mitteldeutschland) hat mindestens 80 Damhirschskelette (D. dama geiselana n. ssp.) und 18 Rothirsche (Cervus elaphus) in den Jahren 1986 bis 1996 geliefert (Abb. 1). Die Funde lassen sich ins Eem (LITT 1994) oder in ein intrasaalezeitliches Interglazial (MANIA 1992, 1996) datieren. Die Skelette sind teilweise vollständig aus gewarvten Seesedimenten geborgen worden. Erstmalig konnte eine fossile Damhirschpopulation hier nach Maßen, morphologischen Merkmalen und bezüglich des Sexualdimorphismus untersucht werden.

D. dama geiselana $\mathrm{n}$. ssp. wird hier beschrieben und aufgrund einer Reihe von morphologischen Eigenmerkmalen am Geweih, der Cervicalwirbelsäule und den Proportionen des Skelettes von den rezenten Unterarten $D$. dama dama und $D$. dama mesopotamica sowie der mittelpleistozänen Unterart $D$. dama clactoniana abgegrenzt. Typische Merkmale der Hirsche von D. dama geiselana sind im Adultstadium eine Schaufellängen-Gesamtlängen-Relation bis zu $75 \%$, besonders lange Augsprossen, schmale, halbmondförmige Geweihschaufeln ohne längere Palmarsprossen und eine breite Dornbildung am Schaufelhinterrand (Abb. 2 bis 4). Die Stange ist bei alten Hirschen unmittelbar über der Augsprosse abgeplattet. Die Linea nucha (Abb. 5) und der Atlas haben eine spezielle Form (Abb. 8). Die oberen Praemolaren weisen dreizackige Sporne auf, ein Polymorphismus besteht am $\mathrm{P}_{4}$ (Abb. 6-7). D. dama geiselana erreicht die Körpergröße von $D$. dama clactoniana und übertrifft die Größe von $D$. dama da$m a$ um 15 bis 20\% (Abb. 9-11). Der Sexualdimorphismus beträgt durchschnittlich 10 bis $15 \%$. Bisher untersuchte Damhirschfunde Deutschlands und Englands aus der Eemwarmzeit erreichen nicht die Maße von $D$. dama geiselana.
\end{abstract}

\section{[The fossil fallow deer of Neumark-Nord (Sachsen-Anhalt, central Germany) - D. dama geiselana n. ssp.]}

Abstract: About 80 skeletons and partial skeletons of a new fossil fallow deer subspecies, $D$. dama geiselana n. ssp. (Cervidae, Artiodactyla) and 18 partial skeletons

\footnotetext{
*) Anschrift der Verfasserin: Dr. Th. Pferffer, Institut für Paläontologie der Universität Bonn, Nußallee 8, 53115 Bonn. Zeichnungen: Verfasserin und R. HAHN, Bonn (Abb. 7), Fundskizzen nach D. Mania, Jena (Abb. 1). Fotos: G. Oleschinski, Bonn.
}

of Cervus elaphus, well preserved, in an articulated state were recovered from the open-cast lignite mine of Neumark-Nord at the northern periphery of the Geiseltal, (SW Halle, Sachsen-Anhalt, central Germany, fig. 1). These finds can be dated as Eemian or as an intra-Saalian interglacial period.

Neumark-Nord represents the first fossil fallow deer population, which is large enough for a statistical and metrical analysis, a morphological description and an analysis of sexualdimorphism. Compared to recent fallow deer, D. dama geiselana described in this paper is characterised by a 15 to $20 \%$ greater body size (figs. 9-11), antlers with up to $75 \%$ palmation, especially long brow tines, and a greater antler spread reminiscent of Megaloceros giganteus. The beam can be flattened above the browtine (figs. 2-4). The linea nucha, the atlas and the upper premolars have a special form, a polymorphism occurs on the $\mathrm{P}_{4}$ (figs. 5-8). The proportions of the limb bones are different from recent Dama dama dama, D. dama mesopotamica and $D$. dama clactoniana. Other finds of Dama dama from Germany and Great Britain dated as Eemian never reach the size of $D$. dama geiselana.

\section{Einleitung}

Aus pleistozänen Seesedimenten von NeumarkNord südwestlich von Halle a. d. Saale (SachsenAnhalt) wurde eine Vielzahl von vollständigen Damhirschskeletten beim Braunkohlentagebau geborgen. Die Damhirsche, die neben Rothirschen, Elefanten, Nashörnern, Auerochsen und einigen Raubtieren die häufigste Säugetiergruppe im Fundmaterial stellen, wurden immer im ähnlichen Erhaltungszustand, mit stark rückgekrümmter Halswirbelsäule gefunden (Abb. 1). D. Mania sowie dem engagierten Einsatz der Mitarbeiter des Braunkohlentagebaus Braunsbedra ist die weitgehend vollständige Bergung dieses außerordentlich bedeutsamen Materials während der Jahre 1986 bis 1996 zu verdanken.

Skelettelemente von mindestens 70 Hirschen und mindestens 10 weiblichen Tieren von D. dama geiselana konnten vermessen werden. Die Einzelreste von D. dama geiselana erhöhen die Mindestindividuenzahl der Damhirsche um minde- 


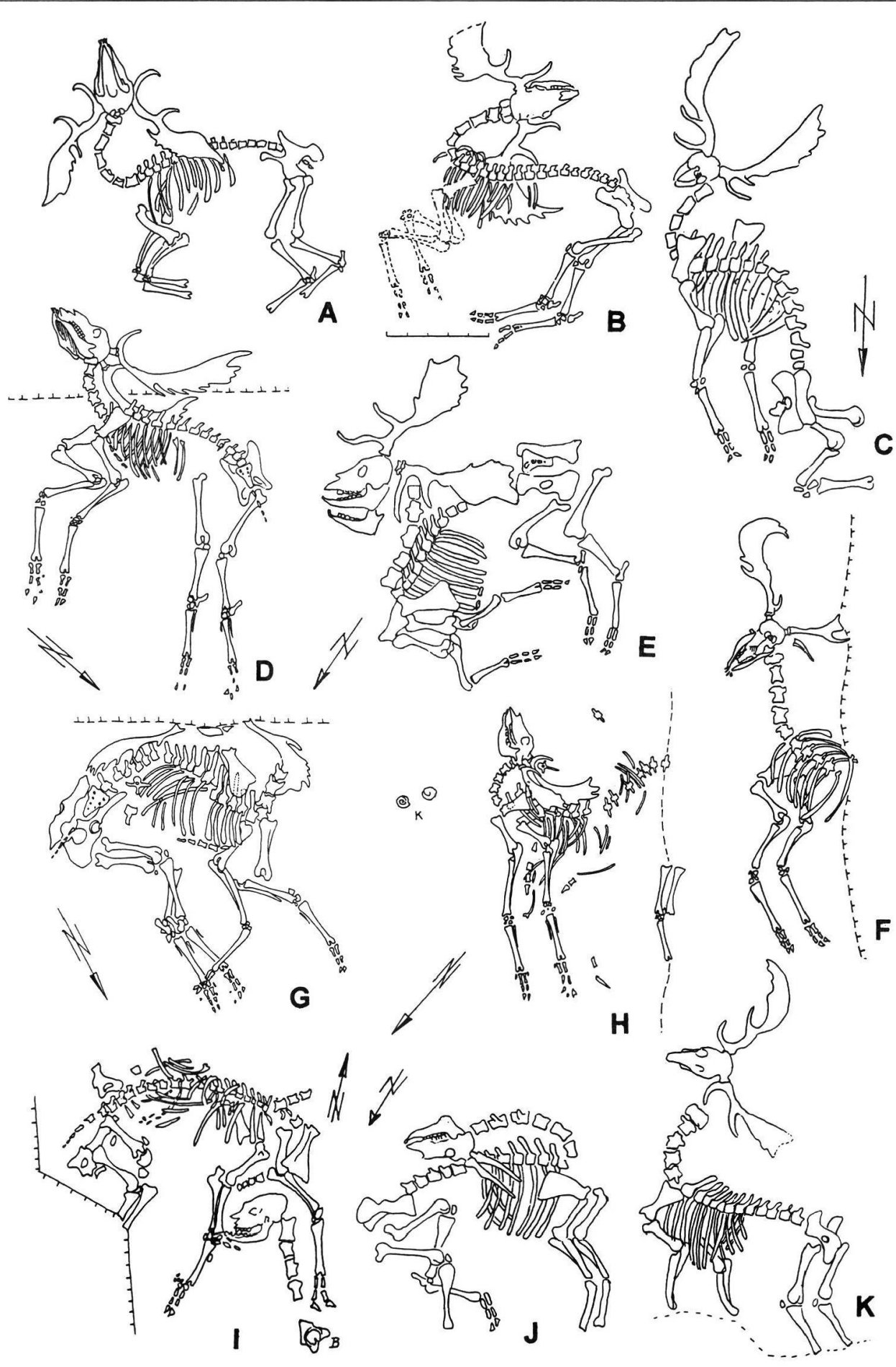

Fig. 1: Skeletons of D. dama geiselana in situ: A-G: Adult stags, C: Holotype: HK 97:14165; H, K: Nearly adult stags; I: Juvenile female; J: Juvenile stag. 
stens 10 und maximal 20. Weibliche Tiere sind stark unterrepräsentiert.

Von den Hirschen sind $17 \%$ juvenil, $22 \%$ subadult (zwei bis fünf Jahre), $44 \%$ adult, meist nur wenig älter als sechs Jahre. Extrem alte Tiere mit einem Individualalter von mehr als 11 Jahren sind nur in zwei Fällen zu verzeichnen. Die Grenzen der Altersstufen lassen sich am Abschluß des Zahnwechsels und der Epiphysenverwachsung der postcranialen Skelettelemente ermitteln. Eine detaillierte Beschreibung der Einzelskelette wird bei PFeIFFer (im Druck, a) gegeben.

Die Funde verteilen sich über die gesamte Fläche des kleinen Sees mit etwa 350 bis 400 m Durchmesser. Vernachlässigt man die Einzelfunde der Uferzone, lassen sich die Cervidenskelette aus Neumark-Nord drei Fundhorizonten zuordnen, wobei die Damhirschfunde im wesentlichen der Feindetritusmudde und der sandigen Grobdetritusmudde entstammen. Ein Überblick wird bei MANIA (1992, 1996), eine detaillierte Darstellung der Fundumstände bei Mania (im Druck) gegeben.

Die zeitliche Einstufung der Funde von NeumarkNord hat Anlaß zu kontroversen Diskussionen gegeben: Während SEIFERT (1990) bei der Pollenanalyse durchaus Parallelen zur Eemwarmzeit feststellt, konstatiert sie andererseits eine große Eigenständigkeit in der Flora des vorliegenden Interglazials. Auch MAI (1990) unterstrich anhand der pflanzlichen Makroreste eine Eigenständigkeit. Nach palynologischen Daten stellt LITT (1994) Neumark-Nord ins Eem. Mania (1992, 1996) plädiert nach Untersuchung der geologischen Verhältnisse und der Mollusken- und Ostracodenfauna für ein intrasaalezeitliches Interglazial. Thermoluminiszenzdaten (ZöLLER, mündliche Mitteilung) weisen auf ein intrasaalezeitliches Interglazial hin.

Aus Neumark-Nord ist soviel Fundmaterial vorhanden, daß erstmalig eine umfangreiche fossile Dama dama-Population bezüglich Körperproportionen, Ontogenese, Sexualdimorphismus und Variabilität untersucht werden konnte. Die Ergebnisse werden von PfEIFFER (im Druck, a) detailliert vorgestellt.

\section{Das Auftreten von Dama im Pleistozän in West- und Mitteleuropa}

Die Gattung Dama Frisch 1775 bezieht sich mit der Typusspecies Dama dama dama auf Schaufelgeweih tragende Hirsche mit Palmarsprossen. Die Stellung von Dama als eigene Gattung war zeitweise strittig, Dama wurde unter der Gattung
Cervus geführt. Erst vor wenigen Jahren konnte sich die Abgrenzung der Gattung Dama von Cervus durchsetzen (Groves \& GrubB 1987, GrubB 1993).

Rezent existieren zwei Unterarten der Art Dama dama, D. dama mesopotamica (BRоOKe 1875), der auf ein kleines Rückzugsgebiet im Iran beschränkt lebt und D. dama dama (LinNÉ 1758), der europäische Damhirsch. Das auffälligste äußere Unterscheidungsmerkmal ist die nur schwache schaufelartige Verbreiterung des Geweihs von $D$. dama mesopotamica, die Schulterhöhe liegt etwa $10 \mathrm{~cm}$ über den Maßen des europäischen Damhirsches (Abb. 4a, d).

Die Dama-Linie läßt sich in Europa bis ins oberste Pliozän zurückverfolgen. Dama rhenana (Dubois 1904) aus Chilhac, Senéze und Tegelen ist als echter Vorfahr Schaufelgeweih tragender Damhirsche zu betrachten, wie PFEIFFER (im Druck, b) an Hand der Analyse postcranialer Skelettmerkmale zeigen konnte. Sie wurden ursprünglich als Cervus rhenanus (DuBoIs 1904, Tegelen) und Cervus philisi (Schaub 1941, Senéze) mit eigenen Artnamen belegt und zur Gattung Cervus im weiteren Sinne gestellt.

Von den mittelpleistozänen Fundstellen Mosbach und Süßenborn ist "Cervus" reichenaui (KAHLKE 1996) bekannt, der in seinen postcranialen Elementen an frühe Damhirsche erinnert (PfEIFFER 1997 a).

Im jüngeren Mittelpleistozän tritt mit Cervus clactonianus (FAlCONER 1868) bzw. Cervus browni (Dawkins 1868) der Fundpunkte Clacton, Swanscombe, Jaywick ein Hirsch mit ansatzweiser Schaufelbildung am Geweih auf. Sutcliffe (1960) sowie Chapman \& CHAPMAN (1975) stellen ihn unter die Gattung Dama. Leonardi \& Petronio (1976) revidieren den eigenen Artstatus und wählen die Bezeichnung D. dama clactoniana. Sie ordnen einen Skelettfund mit gut erhaltenem Geweih aus Riano bei Rom D. dama clactoniana zu. Auffällig sind bei dieser Form der deutlich größere und kräftigere Wuchs gegenüber $D$. dama dama und die Unterschiede am Geweih. Kapitale Hirsche dürften eine Geweihlänge von annähernd 1,20 $\mathrm{m}$ erreicht haben (Abb. $4 \mathrm{~b}$ ).

Für die Eem-Warmzeit werden von vielen Autoren Funde aus dem europäischen Raum beschrieben: Dama dama erreicht sein größtes natürliches Verbreitungsgebiet unter optimalen warmzeitlichen Klimabedingungen. Die europäischen Funde des Eems verzeichnen Koenigswald (1986) und Pfeiffer (1995) detailliert. In den Bereich des letzten Interglazials stellt KoEnigswald (1988) 
auch die Damhirschfunde der nördlichen Oberrheinebene. Sie bleiben in der Größe deutlich hinter D. dama clactoniana zurück, sind aber größer als der rezente europäische Damhirsch und weisen in ihrer Geweihmorphologie Eigenmerkmale auf (PFEIFFER 1997b).

Die Damhirsche von Neumark-Nord zeigen gegenüber $D$. dama clactoniana deutliche morphologische Abweichungen und sind auch gegenüber den rezenten Damhirschen verschieden. Sie werden hier als neue Subspecies von Dama dama beschrieben und erhalten den Namen $D$. dama geiselana. Eemzeitliche Damhirsche Deutschlands sind in der Regel deutlich kleiner (PFeIFFER 1997b). deutlich S- förmig geschwungen (Abb. 5). Charakteristisch ist die Ausbildung von drei kleinen Spornen an der Praehypocrista der oberen Praemolaren (Abb. 6). Eine Molarisierung des P3 tritt nicht auf. Der P4 zeigt einen Trend zur Molarisierung, der letzte Loph des M3 kann mit einer deutlichen Spitze enden.

Die Alae des Atlas sind bei $D$. dama geiselana lateral stets gerade. Die distalen Elemente der Extremitäten sind bei $D$. dama geiselana proportional deutlich länger als bei rezenten Damhirschen.

\subsection{Beschreibung des Holotyps}

Katalog-Nr.: (HK 97:14165, NN 17)

Funddatum: 29.5.1989

\section{D. dama geiselana n. ssp.}

\begin{tabular}{lll}
\hline Familie: & Cervidae & Goldfuss, 1820. Handbuch der Zoologie, 2:xx, 374. \\
Unterfamilie: & Cervinae & Goldfuss, 1820. Handbuch der Zoologie, 2:xx, 374. \\
Gattung: & Dama & Frisch, 1775. Das Natur-System der Vierfüßigen Thiere, 3. \\
Art: & Dama dama (Linndeus, 1758). Syst. Nat.,10. Aufl.,1:67. \\
Unterart: & D. dama geiselana n. ssp. \\
Holotyp: & HK 97: 14165 (NN 17), weitgehend vollständiges Skelett mit rechtem und linkem \\
& Schaufelgeweih eines etwa sieben- bis achtjährigen Damhirsches (Abb. 1c, 2). \\
Funddatum: & 22. Mai 1989 \\
Paratypen: & Die Damhirschskelette und Teilskelette der gleichen Fundstelle. \\
Derivatio nominis: & Benannt nach dem Geiseltal SW von Halle, Sachsen-Anhalt, Deutschland. \\
Locus typicus: & Pleistozänes Seebecken von Neumark-Nord, Geiseltal, Sachsen-Anhalt. \\
Stratum typicum: & Interglaziale Seesedimente, Fein- und Grobdetritusmudde, Algenmudde, nach \\
& LiTT (1994) Eem, nach MaNiA (1992) älter. \\
Aufbewahrungsort: Forschungsstelle Bilzingsleben, Außenstelle der Friedrich Schiller Universität, \\
& Jena, Sachsen-Anhalt. Alle Funde aus Neumark-Nord gehören dem Landes- \\
& museum für Vorgeschichte (Landesamt für archäologische Denkmalpflege) in \\
& Halle/Saale (Land Sachsen-Anhalt) und sind mit Nummern des Hallenser Katalogs \\
& (HK: 97 ...) nach Jahr und fortlaufender Numerierung verzeichnet.
\end{tabular}

\subsection{Diagnose}

Damhirsch, der die Körpergröße von D. dama clactoniana erreicht und die von $D$. dama dama um 15 bis $20 \%$ überschreitet.

Das Geweih zeigt eine beträchtliche Schaufelbildung, die bei erwachsenen Hirschen bis zu $75 \%$ der Gesamtgeweihlänge erreicht (Abb. 2-4). Die Stange ist bei erwachsenen Hirschen bereits im Bereich der Augsprosse lateral abgeflacht, die Augsprosse steht im stumpfen Winkel zur Stange, setzt unmittelbar über der Rose an und ist mit maximal $280 \mathrm{~mm}$ sehr lang. Palmarsprossen fehlen am Geweih von $D$. dama geiselana häufig völlig, die Schaufel ist relativ schmal und abgerundet. Ein abgeflachter Dorn ist palmar in die Schaufel integriert. Die Stellung des Geweihs ist besonders breit ausladend. Die Linea nucha des Hinterhauptes ist
Fundschicht: Mittlere Algenmudde (siehe MANIA 1992).

Erhaltungszustand: Die Knochen sind rehbraun, matt und von fester Beschaffenheit.

Das Skelett konnte in situ dokumentiert werden (Abb. 1c). Die Wirbelsäule war im Thoraxbereich bauchwärts gekrümmt, die Halswirbelsäule aufrecht, im Verband mit dem auf der Stirn liegenden Schädel mit Geweih. Das Skelett ist weitgehend vollständig erhalten, es fehlen ein Metatarsus und die hinteren Phalangen. Durch den Druck des auflastenden Sediments wiesen zahlreiche Knochen Brüche auf. Die Stellung der Vorderbeine war gerade und parallel, die Hinterbeine leicht angewinkelt und etwas nach hinten verlagert. Die günstigen Fundumstände ermöglichten eine spätere, fast vollständige Rekonstruktion des Tieres. 
Bei HK 97:14165 handelt es sich um einen jungen, adulten Damhirsch der nach Geweihentwicklung, Zahnalter und postcranialem Skelett etwa 7 bis 8 Jahre alt geworden sein muß. Einige Thoracalwirbelepiphysen waren noch nicht geschlossen, das Caput humeri bereits fest verwachsen.

Das Geweih ist vollständig. Es zeigt eine altersgemäße Entwicklung, keine Eissprosse, aber bereits einen noch nicht sehr großen Dorn am Hinterrand der rechten und linken Schaufel. Die Augsprossen sind lang, 240 und $230 \mathrm{~mm}$, werden aber noch von Hirsch (HK 97:14159) mit 280 mm übertroffen.

Auffällig ist die starke Beanspruchung und Abnutzung der vorderen Geweihsprossen, sie sind regelrecht blank poliert (Abb. 2). Es handelt sich
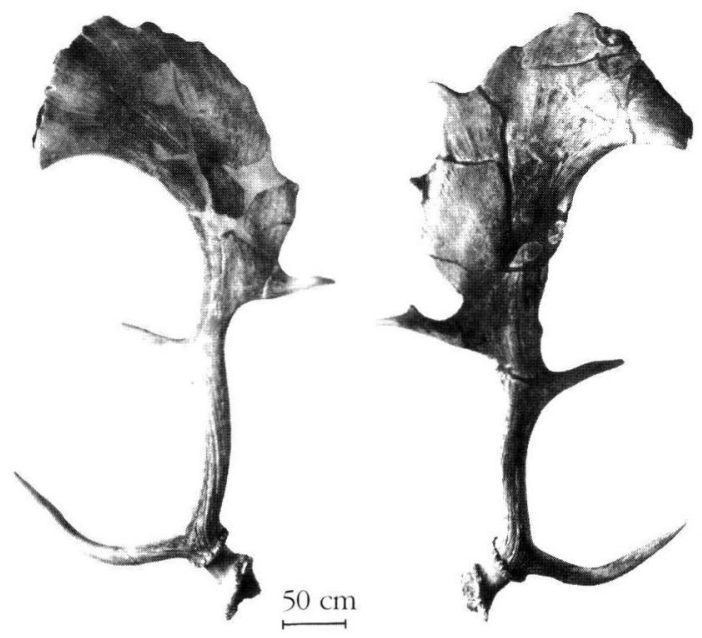

Abb. 2: Rechte und linke Geweihschaufel des Holotyps (HK 97:14165) von D. dama geiselana. Der Hirsch hatte mit 7-8 Jahren Individualalter noch nicht den Höhepunkt der Geweihentwicklung erreicht.

Fig. 2: Right and left antler of the holotype of $D$. dama geiselana (HK 97: 14165). Aged 7 to 8 years, this stag did not reach the optimum of antler development.

also keinesfalls um ein frisch geschobenes Geweih. Der Todeszeitpunkt des Hirsches läßt sich im Kontext mit dem Epiphysenschluß der postcranialen Skelettelemente auf den Herbst bis Winter eingrenzen.

Es handelt sich um einen großen Hirsch der Population von Neumark-Nord. Die Längenmaße seiner Extremitäten markieren meist die obere Grenze, $232 \mathrm{~mm}$ am Humerus, $320 \mathrm{~mm}$ an Radius und Ulna, $243 \mathrm{~mm}$ am Metacarpus III + IV. Diese Werte werden auch von älteren Hirschen der Po- pulation nicht erreicht, obwohl HK 97:14165 noch nicht auf dem Höhepunkt seiner Entwicklung angelangt war. Hirsch (HK 88:12,1-106), Hirsch (HK 88:3,1-86) und (HK 97:14179), die alle älter waren als HK 97:14165, reichen durchschnittlich bis auf $5 \mathrm{~mm}$ an Längenmaße der Extremitätenknochen heran.

\subsection{Metrischer Vergleich und Diskussion 3.3.1 Geweihe}

22 weitgehend vollständige Geweihe von $D$. $d a$ ma geiselana aus Neumark-Nord wurden in die metrische Analyse einbezogen, von 15 Damhirschen konnten beide Geweihschaufeln untersucht werden. Sie wurden mit 27 Geweihen von D. dama dama verglichen. Bei der Beurteilung der Geweihe ist eine deutliche Differenzierung des Individualalters erforderlich.

Eine besondere Bedeutung kommt der Schaufellängen-Gesamtlängen-Relation (GLs/GL) der Geweihstangen zu. Zwei- bis dreijährige Hirsche aus Neumark-Nord mit vollständigem Geweih erreichen eine mittlere Geweihstangenlänge von $256 \mathrm{~mm}$, bei einer Schaufellänge von $221 \mathrm{~mm}$. Das entspricht einer Schaufellängen-Gesamtlängen-Relation von 47\%. Rezente europäische Damhirsche erreichen in diesem Alter lediglich mittlere 37\%. Bei den adulten Hirschen von $D$. dama geiselana nimmt der Anteil der Schaufellänge an der Gesamtlänge erheblich zu. Bei einem Individualalter von 9 Jahren beträgt er bereits $74 \%$ (Abb. 3).

Der Unterschied zu D. dama dama wird an diesem Punkt besonders deutlich, kein untersuchter Hirsch von $D$. dama dama überschritt in der Relation (GLs/GL) den Wert von 59\%.

Dreijährige Hirsche von D. dama geiselana weisen eine halbmondförmige, noch schmale Schaufel auf, größte Breite (GBs) $95 \mathrm{~mm}$, die für die frühe Entwicklungsstufe erstaunlich geschlossen ist und keine langen Palmarsprossen aufweist. Fünfährige Hirsche zeigen mit mittleren 142,5 mm ebenfalls niedrige Werte. Auch bei den adulten Hirschen von $D$. dama geiselana werden lediglich mittlere $178 \mathrm{~mm}$ (GBs) erreicht. Die schmale Schaufel, ohne lang ausgezogene Endsprossen ist ein charakteristisches Merkmal von D. dama geiselana. Rezente europäische Damhirsche bilden selten bereits im 3. Jahr eine geschlossene Schaufel, sie ist oft tief geschlitzt. Bei einem rezenten, vierjährigen Hirsch konnten bereits $250 \mathrm{~mm}$ Schaufelbreite gemessen werden. Durchschnittlich sind die Geweihschaufeln von 

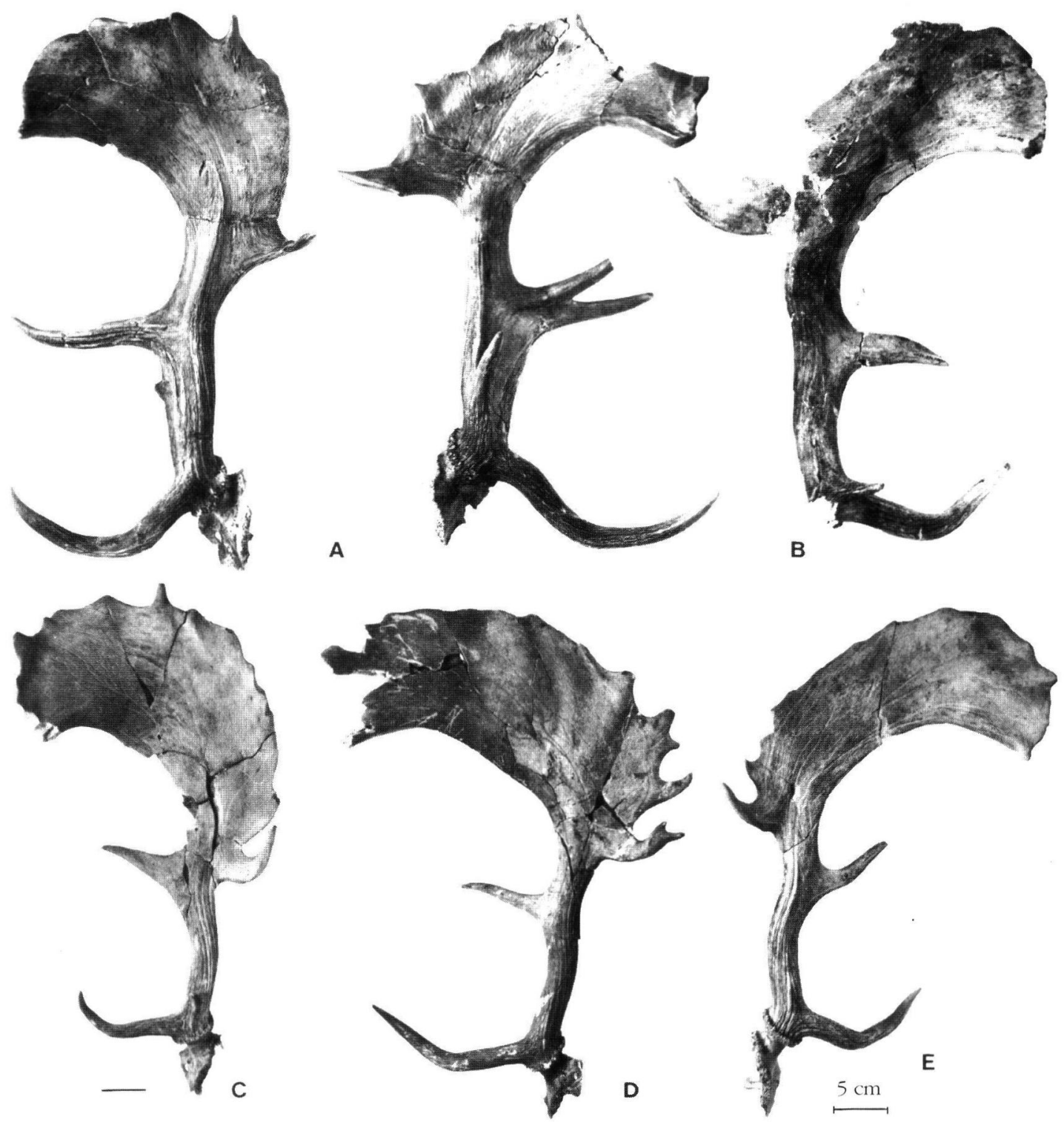

Abb. 3: Geweihschaufeln adulter und subadulter Hirsche von D. dama geiselana. Die Stange ist zum Teil abgeplattet, der Anteil der Schaufel an der Gesamtlänge ist besonders hoch. Die Augsprosse ist besonders lang, die $\mathrm{A}_{2}$ abgeflacht. Palmarsprossen fehlen häufig völlig.
A: NN D61 B: HK 87:300,1089-1113
C: HK 97: 14179 D: HK 87:300, 713-799
E: HK 88:1,1-1,69

Fig. 3: Antlers of adult and nearly adult stags of $D$. dama geiselana. The beam can be flattened, and the percentage of palmation is high in relation to total length. The browtine is especially long, the second anterior tine is flattened. Posterior tines are lacking totally in many cases.
A: NN D61
B: HK 87:300,1089-1113
C: HK 97: 14179
D: HK 87:300, 713-799
E: HK $88: 1,1-1,69$

D. dama dama bei adulten, älter als siebenjährigen Hirschen $252 \mathrm{~mm}$ breit $(\mathrm{n}=21)$.

Die Augsprosse $\left(\mathrm{A}_{1}\right)$ ist bei $D$. dama geiselana besonders lang, schlank und aufrecht gebogen, $280 \mathrm{~mm}$ Länge bilden den Maximalwert. Bereits ein dreijähriger Hirsch weist $185 \mathrm{~mm}$ Augsprossenlänge (LA1) bei einem Durchmesser von 29 $\mathrm{mm}$ auf. Der Mittelwert der dreijährigen Hirsche liegt bei $163 \mathrm{~mm}(\mathrm{n}=8)$. Der Durchschnittswert untersuchter adulter europäischer Damhirsche 

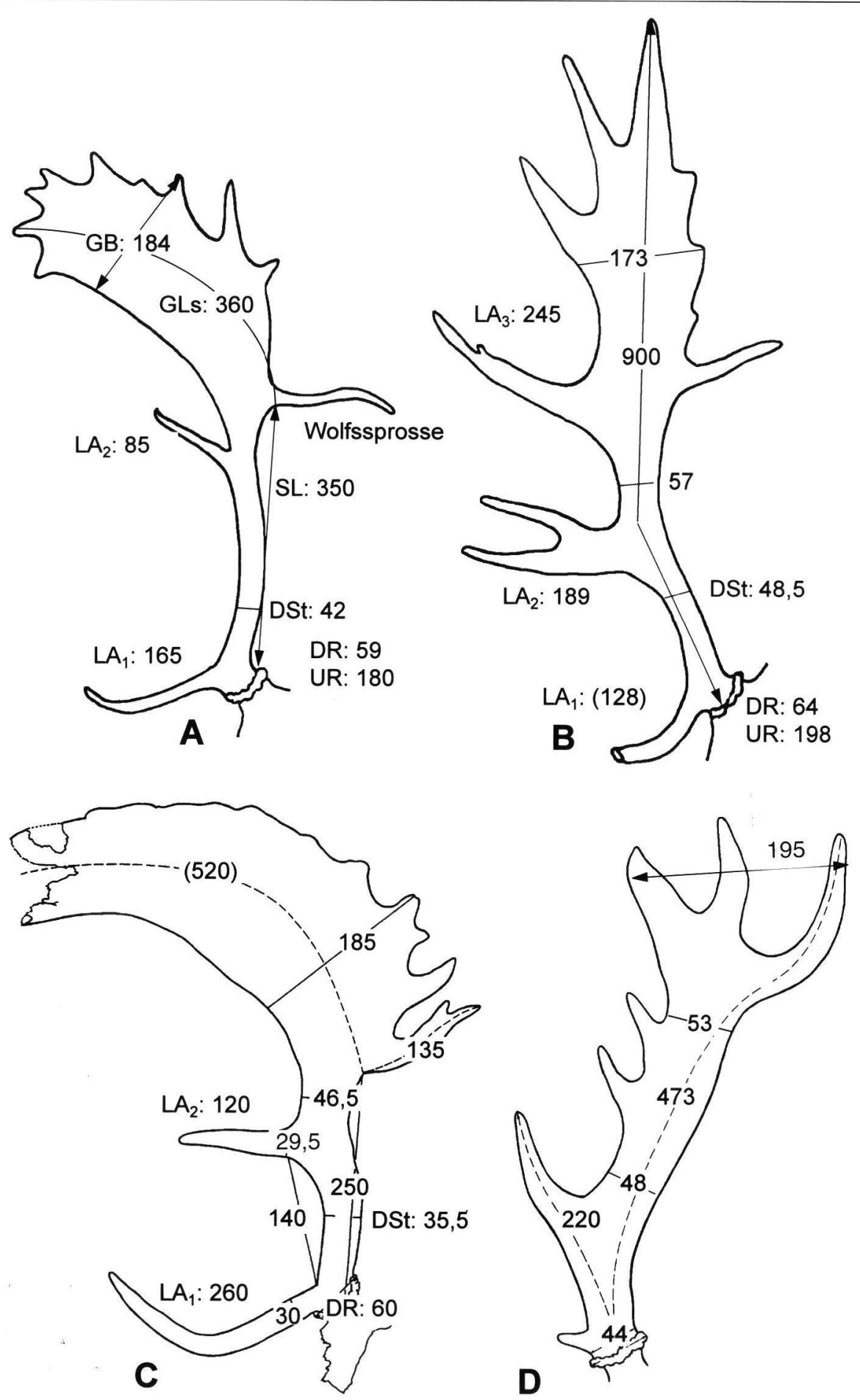

Abb. 4: Schematischer Vergleich der Geweihe von A: D. dama dama; B: D. dama clactoniana (Swanscombe BMNH M16349); C: D. dama geiselana (HK 87:300, 713-799); D: D. dama mesopotamica.

Fig. 4: Schematic comparison of the antlers of A: D. dama dama; B: D. dama clactoniana (Swanscombe BMNH M16349); C: D. dama geiselana (HK87:300, 713-799); D: D. dama mesopotamica. 
liegt mit $141 \mathrm{~mm}$ Augsprossenlänge $(\mathrm{n}=21)$ deutlicher unter den Maßen von D. dama geiselana. Umfang (UR) und Durchmesser (DR) der Rose zeigen dagegen bei $D$. dama geiselana und $D$. dama dama überlappende Streubreiten. Alle Hirsche von $D$. dama geiselana $(n=21)$ weisen $a b$ dem 3. Lebensjahr zunächst noch einen kleinen, mit zunehmendem Lebensalter kräftigen Dorn am hinteren Schaufelrand auf, (Abb. 2). Bei D. dama dama zeigen von den 22 untersuchten Hirschen der Altersgruppe ab fünf Jahre nur zwei eine Dornbildung.

D. dama geiselana setzt sich ebenfalls deutlich in der Geweihentwicklung von $D$. dama clactoniana und $D$. dama mesopotamica ab. Die bei $D$. dama clactoniana häufiger auftretende Gabelung der $\mathrm{A}_{2}$ tritt bei $D$. dama geiselana nur einmal auf (Abb. 3b). Eine Schaufelbildung ist bei den Hirschen aus Clacton nur ansatzweise zu beobachten. Für die Hirsche aus Swanscombe sind die lang ausgezogenen Endsprossen am vorderen und oberen Schaufelrand, sowie lang ausgezogene Sprossen am Hinterrand typisch (Abb. 4b). Für die Geweihe von $D$. dama geiselana ist charakteristisch, daß am vorderen Schaufelrand oberhalb der $\mathrm{A}_{2}$ keine weiteren Sprossen auftreten. Am hinteren Schaufelrand fehlen lang ausgezogene Sprossen, lediglich kurze Sprossen kommen bei den jüngeren Hirschen vor. Die Geweihschaufel wirkt flächig, fast halbmondförmig.

Rezente mesopotamische Damhirsche zeichnen sich durch besonders kurze Geweihe aus, die bei adulten Hirschen zwischen 50 und $70 \mathrm{~cm}$ lang sind und bereits unmittelbar über der Augsprosse flächig verbreitert sind. Sie zeigen keine eigentliche Schaufelbildung, sondern eher eine verbreiterte Stange, die in einer terminalen Endgabel enden kann oder auch viele, abgeplattete Palmarsprossen aufweisen kann (Abb. 4 d). Während bei den pleistozänen Formen allgemein eine Augsprosse vorhanden ist, wie Di Stephano (1996) für mittelpleistozäne mesopotamische Damhirsche aus Tabun E zeigt, ist sie bei rezenten mesopotamischen Damhirschen sehr kurz. Die nach anterior gerichteten Sprossen können bei $D$. dama mesopotamica vielfältige Ausprägungen annehmen. Eine tiefstehende, abgeflachte $\mathrm{A}_{2}$ ist immer vorhanden und übertrifft die $\mathrm{A}_{1}$ an Länge und Stärke. Sie kann von einer Reihe weiterer, abgeflachter Sprossen begleitet werden.

\subsubsection{Occipitalregion}

Die stark geschwungene Linea nucha (Abb. 5) von $D$. dama geiselana tritt bei rezenten Damhirschen nicht in dieser Ausprägung auf. Bei ihnen und auch bei $D$. dama clactoniana wirkt die Linea nucha eher flach, nach lateral breit ausgezogen.

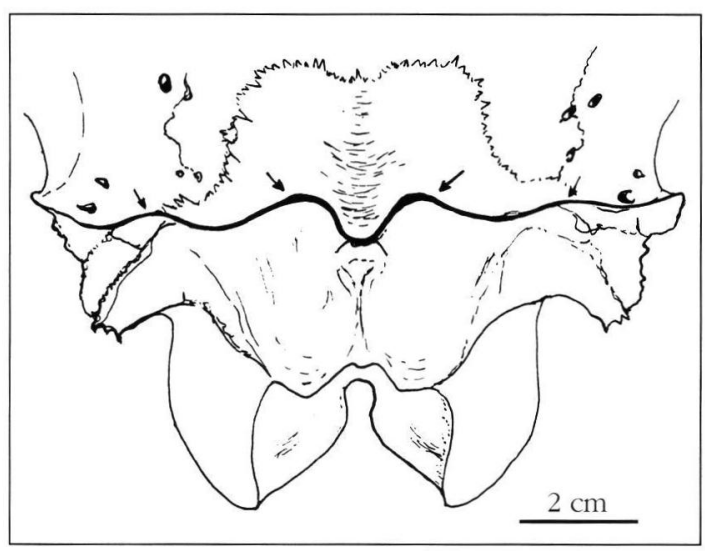

Abb. 5: Linea nucha von D. dama geiselana mit einer deutlich S-förmigen Biegung. Maßstab $2 \mathrm{~cm}$ Fig. 5: Nuchal line of D. dama geiselana exhibits a special S-shape.

\subsubsection{Zähne}

Meßwerte der Zahnreihen von $D$. dama geiselana liegen im Streubereich von $D$. dama clactoniana und überschreiten die Werte von D. dama dama um 15 bis 20\%. Der Sexualdimorphismus ist mit etwa 5\% gering. Eine morphologische Besonderheit der Damhirsche aus Neumark-Nord sind drei Sporne, die durchgängig an der Praehypocrista der oberen Praemolaren auftreten (Abb. 6). Dieses Merkmal hat eingeschränkten diagnostischen Wert für $D$. dama geiselana, es konnte bei $D$. dama clactoniana nicht beobachtet werden, bei D. dama dama in drei Fällen von 67 und bei pleistozänen mesopotamischen Damhirschen aus Kebara (Israel) in 6 von 39 Fällen.

Die Zahnreihen von $D$. dama geiselana erreichen durchschnittlich fast $100 \mathrm{~mm}$, die der rezenten Damhirsche etwa $88,5 \mathrm{~mm}$. Der $\mathrm{M}_{3}$ der Hirsche von $D$. dama geiselana ist wie bei $D$. dama clactoniana durchschnittlich etwas mehr als $25 \mathrm{~mm}$ lang, während rezente Damhirsche durchschnittlich etwas mehr als $22,5 \mathrm{~mm}$ aufweisen.

Ein Polymorphismus zeigt sich am $\mathrm{P}_{4}$ von $D$. $d a$ ma geiselana. Hier wird ein unterschiedlicher Grad der Molarisierung erreicht (Abb. 7).

\subsubsection{Atlas}

Bedingt durch die beträchtliche Geweihentwicklung der Damhirsche ist vor allem die Breite des Atlas starkem ontogentischen Wachstum unterworfen, der Sexualdimorphismus ist erheblich. 

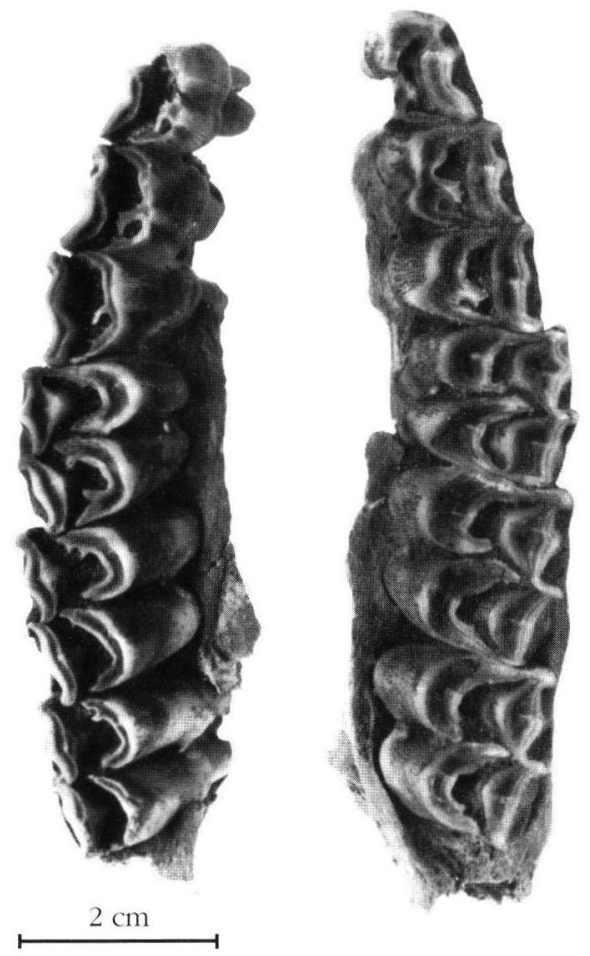

Abb. 6: Oberkieferzahnreihen von D. dama geiselana mit dreifach gezackten Spornen der Praemolaren.

links: HK 88: 2,6 (3 Jahre); rechts: HK 97: 14165 (Holotyp, ca. 7 Jahre).

Fig: 6: Maxillary tooth rows of $D$. dama geiselana showing special characters on the premolars.

left: HK 88: 2,6 (3 years old); right: HK 9714165 (Holotype, ca. 7 years).

Bei adulten Hirschen, mit nachweislich starkem Geweih können die Breitenmaße des Atlas die der Weibchen um 25\% überschreiten. Für adulte Hirsche läßt sich für die untersuchten Dama dama- Unterarten eine charakteristische Form der Alae des Atlas feststellen (Abb. 8).

D. dama dama und D. dama mesopotamica zeigen lateral eine konkave Einbuchtung der Alae, bei D. dama clactoniana sind die Alae konvex gebogen, cranial schmaler als caudal und bei $D$. dama geiselana gerade. Die Atlasform kann als diagnostisches Merkmal der Subspecies von $D a$ ma dama gewertet werden.

\subsubsection{Extremitäten}

In den Meßwerten der Langknochen überschreitet $D$. dama geiselana rezente europäische Damhirsche deutlich um etwa 15\%. Dabei ist ein erheblicher Sexualdimorphismus von 15 bis 20\% bei rezenten europäischen Damhirschen und von

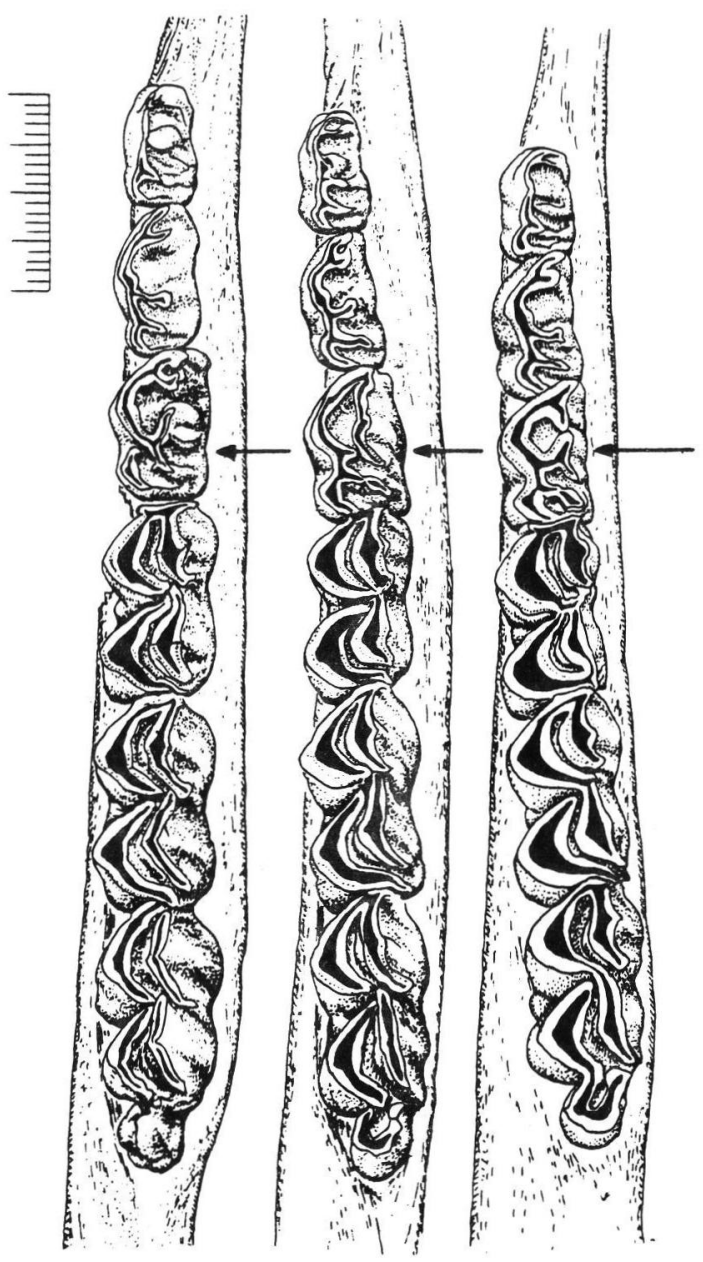

Abb. 7: Unterkieferzahnreihen von $D$. dama geiselana. Es zeigt sich ein Polymorphismus am $\mathrm{P}_{4}$. links: HK 88: 2,6, Mitte: HK 97: 14170; rechts: HK 97: 14162 .

Fig. 7: Mandibular tooth rows of $D$. dama geiselana with a polymorphic $\mathrm{P}_{4}$

left: HK 88: 2,6; middle: HK 97: 14170; right: HK 97: 14162.

10 bis $15 \%$ bei $D$. dama geiselana zu berücksichtigen. Starke Hirsche von D. dama dama erreichen somit den Streubereich der Weibchen aus Neumark-Nord (Abb. 9, 10). Tab. 1 gibt einen Überblick über den Streubereich der Meßdaten an den Langknochen von $D$. dama geiselana.

Bilden die Meßwerte im Längen-Breiten-Diagramm an den Langknochen bei Männchen und Weibchen von Dama dama stets getrennte Punktwolken, gilt dies nicht für Metacarpus und Metatarsus. Sowohl bei D. dama dama als auch 


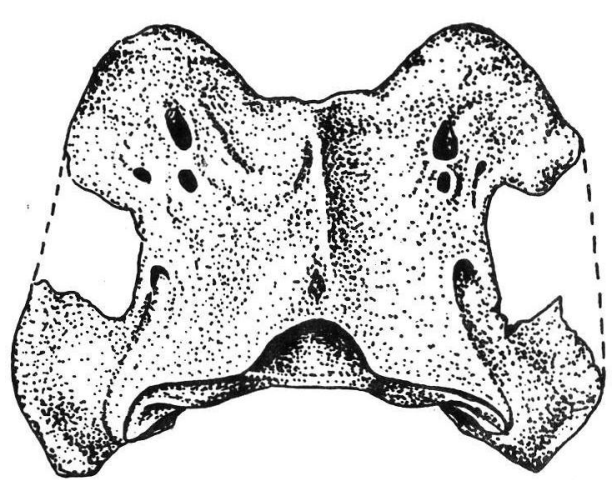

A

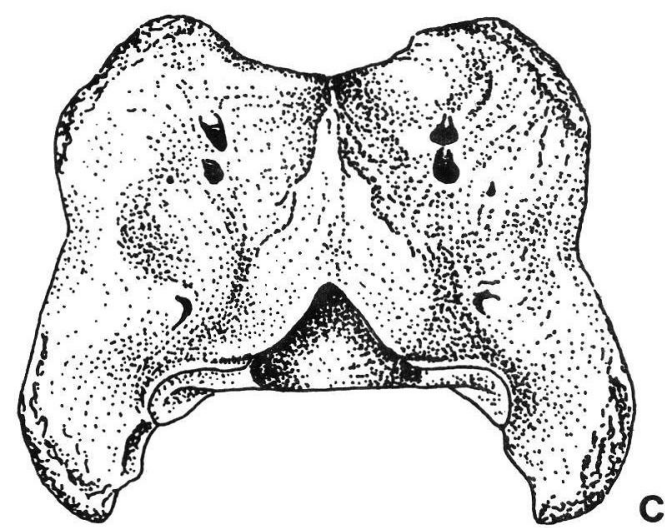

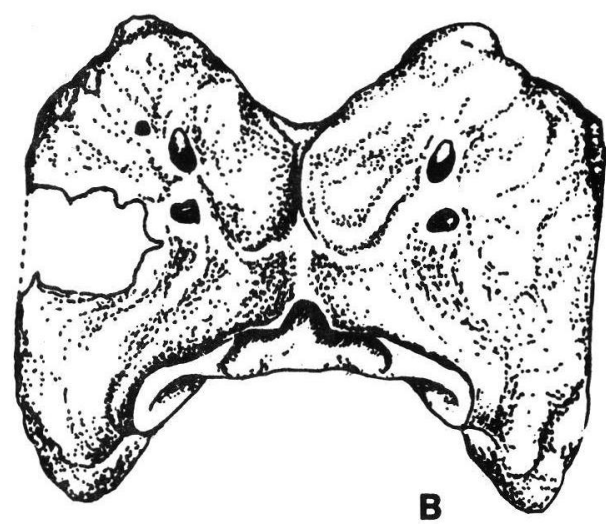

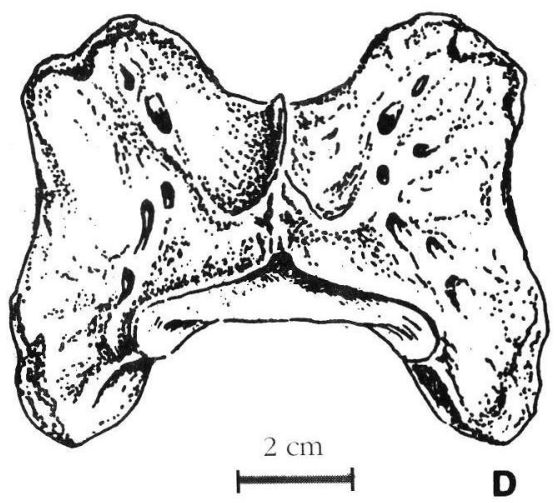

Abb. 8: Atlasformen von A: D. dama clactoniana (Clacton, BMNH M28037); B: D. dama geiselana (HK 97: 14159); C: D. dama mesopotamica; D: D. dama dama.

Fig. 8: Atlas of A: D. dama clactoniana (Clacton, BMNH M28037); B: D. dama geiselana (HK 97: 14159); C: D. dama mesopotamica; $\mathrm{D}:$ D. dama dama.

bei $D$. dama geiselana sind die Metapodien der Weibchen im Mittel nur 5\% kürzer als bei den Damhirschen, die Diaphysen sind auffallend schlank.

D. dama clactoniana aus dem jüngeren Mittelpleistozän erreicht die Größe von D. dama geiselana, zeigt aber unterschiedliche Extremitätenproportionen.

Aus Clacton und Swanscombe liegt kein vollständiger Humerus vor, distale Breitenmaße entsprechen $D$. dama geiselana. Auch die Radiusmaße von $D$. dama clactoniana liegen im Streufeld von D. dama geiselana, während die Metacarpuslängen etwas geringer ausfallen. Die Längenmaße der 1 . Phalanx anterior können bei $D$. dama clactoniana geringfügig höher sein (Abb. 9).

Deutlicher sind die Proportionsunterschiede an der Hinterextremität (Abb. 10). D. dama clactoniana zeigt in der Regel einen längeren Femur und Calcaneus als D. dama geiselana, während D. dama geiselana längere Tibia- und Metatarsusmaße aufweist.

Die Betonung der distalen Elemente der Hinterextremität bei $D$. dama geiselana spricht für eine Anpassung an schnelles Laufen. Bei D. dama geiselana dürfte es sich um einen flinken Hirsch mit langen, schlanken Extremitäten und hoher Wendigkeit gehandelt haben.

D. dama clactoniana mag wegen der besonders langen Geweihe und den stabiler gebauten Extremitäten einen etwas gedrungeneren Körperbau gehabt haben. Gegen die mittelpleistozänen Damhirsche wirkt der kleine rezente europäische Damhirsch eher plump mit kurzen Beinen (Abb. 11) und großem Geweih. Hier mag menschliche Selektion zugunsten großer Trophäen bei gleichzeitig fehlendem Druck durch große Fleischfresser Ursache einer starken Verkürzung der Lang- 
Tab. 1: Auswertung der Extremitätenmaße von D. dama geiselana. Meßstrecken nach v. d. Driesch (1976).

Tab. 1: Dimensions of limb bones of D. dama geiselana. Measurement of v. d. Driesch (1976).

\begin{tabular}{|c|c|c|c|c|c|c|c|c|c|c|c|c|c|}
\hline \multirow{2}{*}{\multicolumn{2}{|c|}{ Humerus }} & $\mathrm{GL}$ & $\mathrm{BP}$ & $\overline{B D}$ & $\mathrm{KD}$ & & & & & $\overline{G L}$ & $B P$ & $\mathrm{BD}$ & $\mathrm{KD}$ \\
\hline & & $n=29$ & $n=35$ & $n=48$ & $n=45$ & & & \multicolumn{2}{|c|}{ Femur } & $n=27$ & $n=35$ & $n=41$ & $n=43$ \\
\hline \multirow{5}{*}{$\sigma^{\circ}$} & Max & 232,00 & 66,00 & 55,00 & 25,00 & & & \multirow{5}{*}{$\sigma^{\circ}$} & Max & 292 & 81 & 67 & 26 \\
\hline & Min & 217,00 & 60,00 & 45,50 & 19,00 & & & & Min & 270 & 71 & 61 & 22 \\
\hline & Mittelw. & 224,45 & 63,63 & 49,05 & 21,92 & & & & Mittelw. & 279,54 & 74,58 & 62,36 & 23,45 \\
\hline & Varianz & 17,97 & 3,18 & 4,41 & 1,33 & & & & Varianz & 39,46 & 6,82 & 1,81 & 1,01 \\
\hline & Standardabw. & 4,24 & 1,78 & 2,10 & 1,15 & & & & Standardabw. & 6,28 & 2,61 & 1,34 & 1,00 \\
\hline & & $n=5$ & $n=6$ & $n=7$ & $n=7$ & & & & & $n=3$ & $n=4$ & $n=4$ & $n=4$ \\
\hline \multirow{4}{*}{$q$} & Max & 209,00 & 55,00 & 46,00 & 19,00 & & & \multirow{4}{*}{ f } & Max & 263 & 69 & 59 & 21 \\
\hline & Min & 175,00 & 45,00 & 43,00 & 16,00 & & & & Min & 260 & 67 & 58 & 21 \\
\hline & Mittelw. & 190,00 & 51,00 & 43,71 & 18,29 & & & & Mittelw. & 262,00 & 67,75 & 58,67 & 21,00 \\
\hline & & GL & $\mathrm{BP}$ & GLr & $\mathrm{BD}$ & $\mathrm{TPa}$ & $\mathrm{KD}$ & & & GL & $\mathrm{BP}$ & $\mathrm{BD}$ & $\mathrm{KD}$ \\
\hline Ra: & lius und Ulna & $n=17$ & $n=37$ & $n=31$ & $n=29$ & $n=29$ & $n=38$ & Tib & & $n=37$ & $n=34$ & $n=43$ & $n=42$ \\
\hline \multirow{6}{*}{ o } & Max & 320,00 & 53,00 & 258,00 & 48,00 & 48,00 & 29,00 & \multirow{5}{*}{ 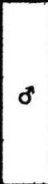 } & Max & 337 & 72 & 45 & 28 \\
\hline & Min & 285,00 & 43,00 & 228,00 & 39,00 & 39,00 & 24,00 & & Min & 305 & 59 & 35,5 & 24 \\
\hline & Mittelw. & 305,35 & 47,68 & 242,48 & 42,19 & 43,47 & 26,28 & & Mittelw. & 319,25 & 66,49 & 41,07 & 25,83 \\
\hline & Varianz & 87,37 & 2,86 & 51,99 & 5,76 & 5,18 & 1,63 & & Varianz & 61,74 & 3,99 & 2,47 & 1,50 \\
\hline & Standardabw. & 9,35 & 1,69 & 7,21 & 2,40 & 2,28 & 1,28 & & Standardabw. & 7,86 & 2,00 & 1,57 & 1,22 \\
\hline & & $n=5$ & $n=8$ & $n=7$ & $n=8$ & $n=5$ & $n=9$ & & & $n=5$ & $n=7$ & $n=8$ & $n=9$ \\
\hline \multirow{4}{*}{$q$} & Max & 283,00 & 44,00 & 229,00 & 39,00 & 38,00 & 23,00 & \multirow{4}{*}{$q$} & $\operatorname{Max}$ & 301 & 64 & 40 & 24 \\
\hline & Min & 231,00 & 40,00 & 182,00 & 36,00 & 34,00 & 19,00 & & Min & 286 & 59 & 37 & 22 \\
\hline & Mittelw. & 255,80 & 42,38 & 205,00 & 37,50 & 35,40 & 21,33 & & Mittelw. & 295,60 & 61,29 & 37,88 & 23,17 \\
\hline & & $\mathrm{GL}$ & $B P$ & $\mathrm{BD}$ & $\mathrm{KD}$ & & & & & $G L$ & BP & $\mathrm{BD}$ & $\mathrm{KD}$ \\
\hline \multicolumn{2}{|c|}{ Metacarpus III+IV } & $n=32$ & $n=33$ & $n=35$ & $n=35$ & & & \multicolumn{2}{|c|}{ Metatarsus III+IV } & $n=29$ & $n=32$ & $n=32$ & $n=35$ \\
\hline \multirow{6}{*}{$\sigma^{\circ}$} & Max & 243,00 & 37,00 & 37,00 & 23,00 & & & \multirow{5}{*}{$\sigma^{\circ}$} & $\operatorname{Max}$ & 270,00 & 36,00 & 37,50 & 22,00 \\
\hline & Min & 227,00 & 33,00 & 32,00 & 19,00 & & & & Min & 252,00 & 29,00 & 34,00 & 18,00 \\
\hline & Mittelw. & 233,47 & 34,28 & 34,57 & 21,00 & & & & Mittelw. & 260,00 & 32,05 & 36,03 & 20,77 \\
\hline & Varianz & 25,21 & 1,10 & 1,56 & 0,89 & & & & Varianz & 21,33 & 1,97 & 0,77 & 1,17 \\
\hline & Standardabw. & 5,02 & 1,05 & 1,25 & 0,95 & & & & Standardabw. & 4,62 & 1,40 & 0,88 & 1,08 \\
\hline & & $n=7$ & $n=7$ & $n=9$ & $n=9$ & & & & & $n=11$ & $n=11$ & $n=12$ & $n=13$ \\
\hline \multirow{5}{*}{$q$} & Max & 224,00 & 33,50 & 33,00 & 20,50 & & & \multirow{5}{*}{ q } & Max & 249,00 & 33,00 & 35,00 & 23,00 \\
\hline & Min & 210,00 & 30,00 & 30,00 & 17,00 & & & & Min & $235, \infty 0$ & 28,00 & 31,00 & 18,00 \\
\hline & Miltelw. & 218,29 & 31,07 & 31,67 & 18,50 & & & & Mittelw. & 242,80 & 29,90 & 33,58 & 19,77 \\
\hline & Varianz & 33,57 & 1,70 & 1,00 & 1,50 & & & & Varianz & 25,73 & 1,66 & 1,54 & 1,69 \\
\hline & Standardabw. & 5,79 & 1,30 & 1,00 & 1,22 & & & & Standardabw. & 5,07 & 1,29 & 1,24 & 1,30 \\
\hline
\end{tabular}

Meßstrecken nach v. d. DRIESCH (1976)

knochen bei gleichzeitig starken Diaphysen sein. Auch der rezente mesopotamische Damhirsch hat kurze und gedrungene Metapodien im Gegensatz zu D. dama geiselana, wenn er auch sonst bis auf 5\% die Maße der mittelpleistozänen Damhirsche erreicht.

Die Eigenständigkeit von $D$. dama geiselana wird nicht nur durch den schlanken und langen Wuchs der Extremitäten deutlich, sondern auch die morphologische Ausprägung der Geweihe, der Linea nucha und der Atlasform spricht für die Abgrenzung dieser Population als neue, fossile Unterart von Dama dama.

\section{Danksagung}

An dieser Stelle möchte ich Prof. W. v. KoenigswalD für die Themenstellung und Betreuung meiner Diplomarbeit, die die Basis dieser Publikation bildete, danken. Herrn Prof. D. MaNia danke ich für Bereitstellung des Skelettmaterials, Aufzeichnungen zum Fundort und Skizzen zur Fundlage der Hirsche. Es ergab sich eine fruchtbare Zusammenarbeit während der letzten Jahre.

Umfangreiches Vergleichsmaterial von rezenten Cerviden wurde vom Institut für Haustierkunde der Universität Kiel, vom Tierkundemuseum in Dresden, vom Zoologischen Museum und For- 


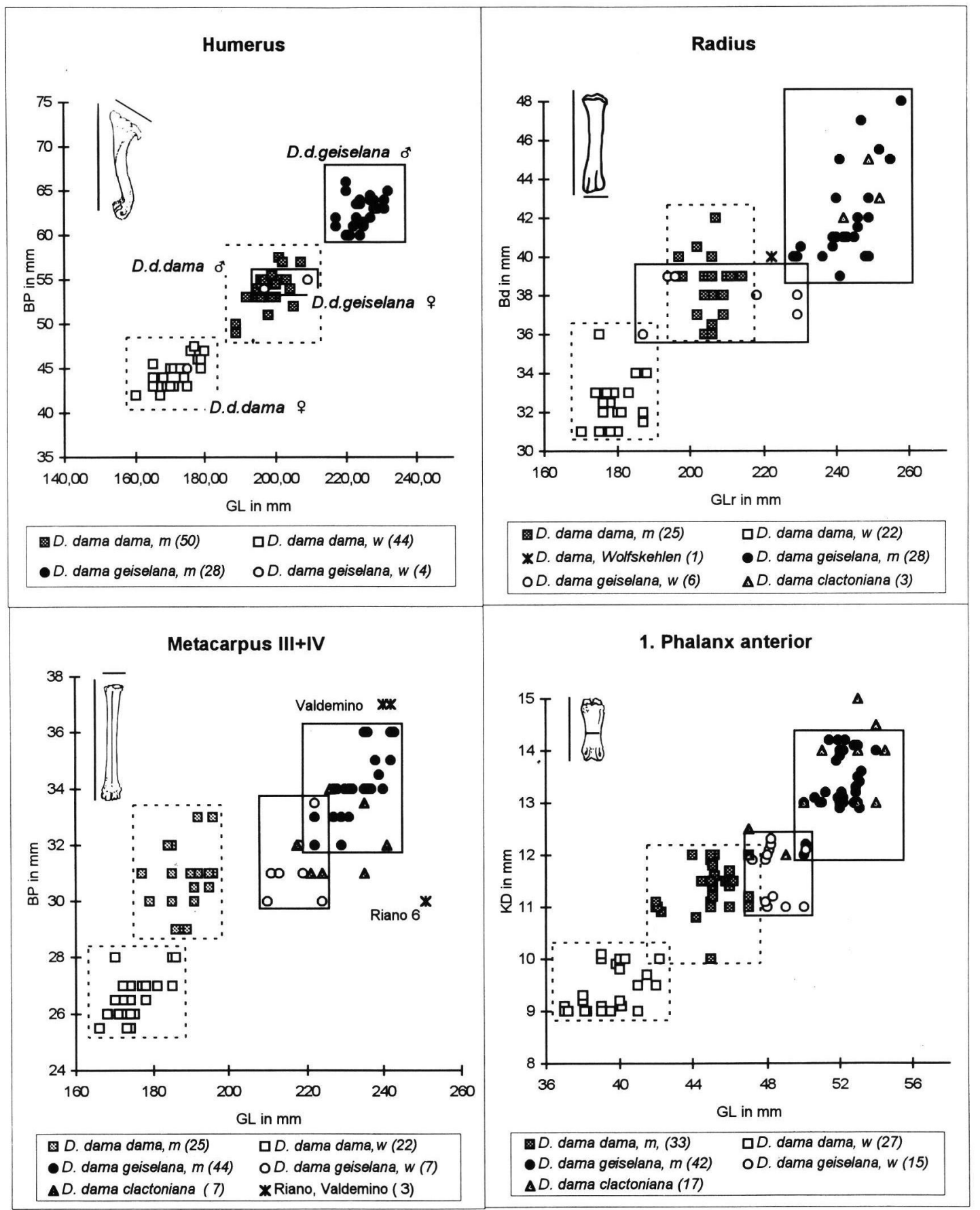

Abb. 9: Längen-Breiten-Relationen an ausgewählten Skelettelementen der Vorderextremität rezenter und fossiler Damhirsche.

$D$. dama geiselana und $D$. dama clactoniana übertreffen rezente und eemzeitliche Damhirsche deutlich in den Extremitätenmaßen.

Fig. 9: Length/width ratios in selected front limb elements of recent and fossil fallow deer.

$D$. dama geiselana and $D$. dama clactoniana clearly exceed recent and Eemian fallow deer in their dimensions. 


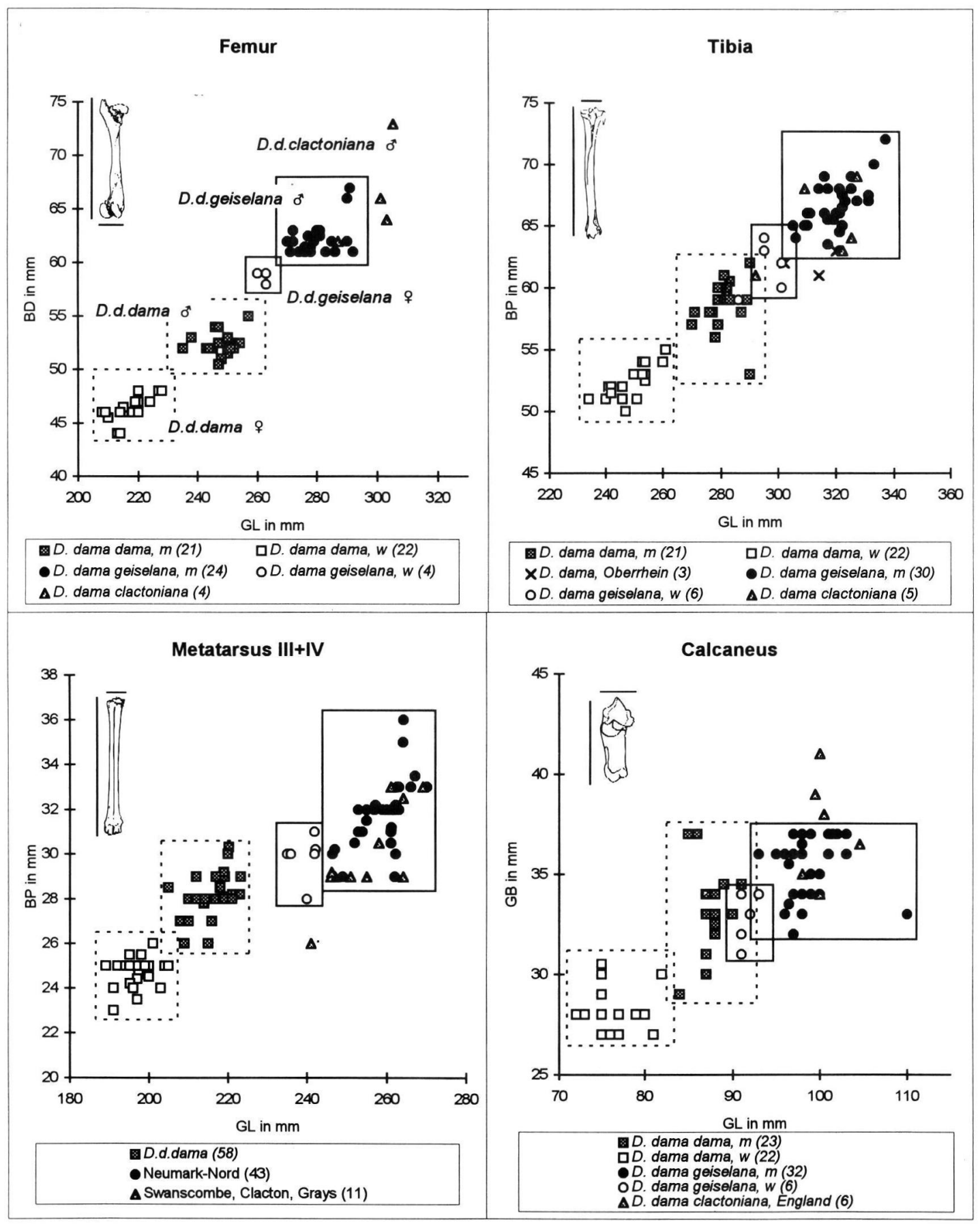

Abb. 10: Längen-Breiten-Relationen an ausgewählten Skelettelementen der Hinterextremität rezenter und fossiler Damhirsche.

D. dama geiselana und $D$. dama clactoniana übertreffen rezente und eemzeitliche Damhirsche deutlich in den Maßen. D. dama geiselana und D. dama clactoniana unterscheiden sich jedoch in den Proportionen der Hinterextremität.

Fig. 10: Length/width ratios in selected hind limb elements of recent and fossil fallow deer.

$D$. dama geiselana and $D$. dama clactoniana clearly exceed recent and Eemian fallow deer in their dimensions, but $D$. dama geiselana and $D$. dama clactoniana show different proportions in the hind limb. 


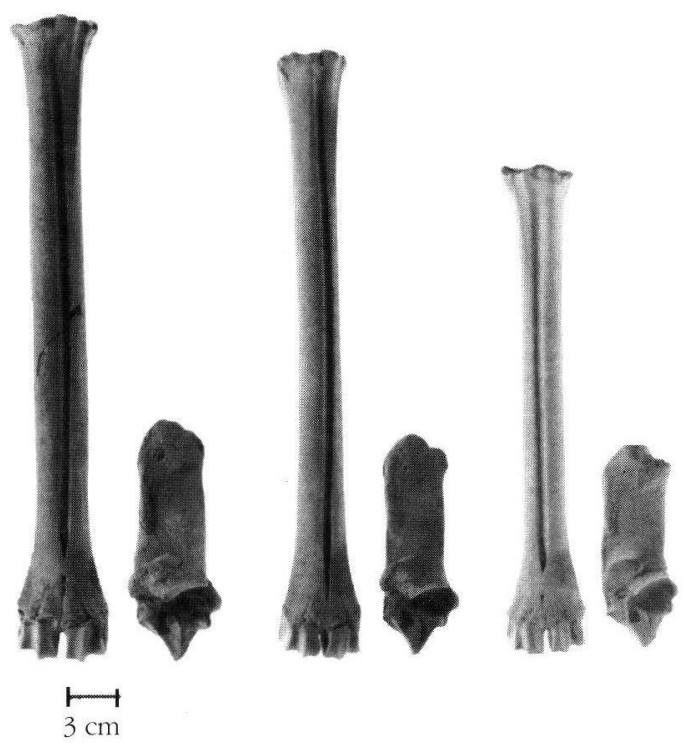

Abb. 11: Metatarsus und Calcaneus von D. dama geiselana und D. dama dama im Vergleich:

links: D. dama geiselana ठे Mitte: $D$. dama geiselana $q$ rechts: D. dama dama $\delta$

Fig:11: Metatarsus and Calcaneus of $D$. dama geiselana in comparison to $D$. dama ddma:

left: $D$. dama geiselana ot middle: $D$. dama geiselana + right: $D$. dama dama ${ }^{\circ}$

schungsinstitut Alexander KoenIG, Bonn, vom Naturkundemuseum in Berlin, vom Senckenbergmuseum in Frankfurt und vom Institut für Paläoanatomie der Universität München zur Verfügung gestellt. Den Herren Dr. H. Reichstein, Dr. G. HeIDemann und Dr. D. Heinrich aus Kiel, Dr. A. Feiler aus Dresden, Dr. R. Hutterer aus Bonn, Frau Dr. R. Angermann aus Berlin, Dr. G. Storch aus Frankfurt, Frau Prof. A. v. d. Driesch aus München sei hier gedankt.

Fossiles Cervidenmaterial des Historischen Museums der Stadt Verden (Lehringen), des Museum of Natural History, London (Swanscombe, Clacton, Jaywick, Trafalgar Square, Joint Mitnor Cave), des Geologischen Instituts der Universität Rom „La Sapienza“ (Riano) konnten in die vergleichende Analyse einbezogen werden. Für die Bereitstellung des Materials und manch informatives Gespräch danke ich Frau Dipl. Prähist. F. Wöbse (Verden), den Herren Dr. A. Sutcliffe, Dr. J. HooKer, Dr. A. CurRant (London), Herrn Dr. G. DI StePHANO und besonders herzlich Prof. Dr. C. PETRONIO und Frau Dr. L. CAPASSO-BABATO (Rom).

Den Präparatoren der Forschungsstelle Bilzingsleben, Frau A. Hitzel und Herrn T. Teich und des Museums Alexander Koenig in Bonn, Herrn
Meurer und Herrn Koplek des Paläontologischen Instituts der Universität Bonn, Herrn W. GEBHARDT danke ich für Hilfe bei der Rekonstruktion einiger stark beschädigter Skelette und Geweihe. Die Zeichnungen der Zahnreihen wurden von Herrn R. HAHN, Universität Bonn ausgeführt. Ich danke Herrn G. Oleschinski, Institut für Paläontologie der Universität Bonn für die ausgezeichneten Fotos.

\section{Schriftenverzeichnis}

Chapman, D. \& Chapman, N. (1975): Fallow Deer: Their history, distribution and biology. - Lavanham and Suffolk.

DAwkins, B. (1887): Monograph on the British Mammalia of the Pleistocene period. Order Ungulata (Artiodactyla, Cervidae) 1887. - Paleont. Soc. 40: (1886) 27 S., 7 Taf.; London.

Di Stefano, G. (1996): The Mesopotamian fallow deer (Dama, Artiodactyla) in the Middle East Pleistocene. - N. Jb. Geol. Paläont. Abh. 199/2: 295-322, 12 Abb.; Stuttgart.

Driesch, A. v. d. (1976): Das Vermessen von Tierknochen aus vor- und frühgeschichtlichen Siedlungen. - Inst. f. Paläoanatomie, Domestikationsforsch. u. Geschichte d. Tiermedizin d. Univ. München: 1-27; München

Duiors, E. (1904): On an equivalent of the Cromer Forest-bed in the Netherlands. - Kon. Akad. Wetensch. Proc. Sect. Sci. 7/3: 214-222; Amsterdam.

FalCONER, H. (1868): Palaeontological memoirs and notes. - 2 Bd., XIII + 67 S., Taf. I - XXXVIII; London (E. Hartwicke).

Groves, C. P. \& GrubB, P. (1987): Relationship of living deer. - In: C. M. Wemmer, ed.): Biology and management of the cervidae, 21-59; Washington D.C. (Smithonian Institution Press).

GrubB, P. (1993): Mammal species of the world: 384387, Washington D.C. (Smithonian Institution Press).

Kalke, H.-D. (1996): Der „kleine Hirsch“ aus dem unteren Mittelpleistozän von Mosbach (WiesbadenBiebrich). - Beitr. z. Geol. Thür., Neue Folge 2: 97-100.

Koenigswald, W. v. (1986): Zur Gliederung des Quartärs. - In: Niethammer, J. \& Krapp, F. (Hrsg.): Handbuch der Säugetiere Europas 2/1: XV-XVII + 149151; Wiesbaden (Akad. Verlagsges.).

- (1988): Palökologische Aussage letztinterglazialer Säugetiere aus der nördlichen Oberrheinebene. In: Koenigswald, W. v. (Hrsg.): Paläoklimaforschung 4: 205-314, 51 Abb.; Stuttgart (Fischer).

Leonardi, G. \& Petronio, C. (1976): The fallow deer of European Pleistocene. - Geologica Romana, 25: 1-67, 56 Abb., 8 Taf.; Rom.

Lister, A. M. (1986): New Results on Deer from Swanscombe, and the Stratigraphical Significance in the Middle and Upper Pleistocene of Europe. - Journ. of. Arch. Science, 13: 319-338, 4 Abb.; London. 
LITT, T. (1994): Paläoökologie, Paläobotanik und Stratigraphie des Jungquartärs im mitteleuropäischen Tiefland. - Dissertationes Botanicae 227: 185 S., 50 Abb., 10Tab., 4 Beil.; Berlin, Stuttgart (Cramer).

MAI, H. D. (1990): Zur Flora des Interglazials von Neumark-Nord, Kr. Merseburg. - Veröff. Landesmus. Vorgesch. Halle 43: 159-160; Halle.

Mania, D. (1990): Das Mittelpaläolithikum von Neumark.Nord - eine besondere ökologisch-ökonomische Fazies. - EAZ 1: 16-24; Berlin.

- (1992): Neumark- Nord ein fossilreiches Interglazial im Geiseltal. - Cranium 9/2: 53-76; Leiden.

- (1996): Das Interglazial von Neumark-Nord (Geiseltal) - Zum Untersuchungsstand 1994. Tübinger Monogr. Z. Urgesch. 11: 217-229; Tübingen.

Pfeiffer, T. (1995): Das Vorkommen von Dama dama in Mitteleuropa im Pleistozän unter besonderer Berücksichtigung der Funde von Neumark-Nord. Z. Jagdwiss. 41: 157-170, 18 Abb.; Berlin (Blackwell Wissenschafts-Verlag).

- (1997a): Dama (Pseudodama) reichenaui (Kahlke 1996) aus den Mosbach-Sanden (Wiesbaden-Biebrich). - Mainzer Naturwissenschaftliches Archiv 35: 31-59, 14.Abb., 2 Tab.; Mainz.

- (1997b): Die fossilen Damhirsche der Oberrheinebene im Vergleich zu Dama dama aus NeumarkNord (Sachsen-Anhalt), Lehringen (Niedersachsen) und dem rezenten europäischen Damhirsch. - Z Jagdwiss. 43: 221-239, 20 Abb., 2 Tab.; Berlin (Blackwell Wissenschafts-Verlag).
- (im Druck, a): Die pleistozänen Hirsche von Neumark-Nord (Sachsen-Anhalt) D. dama geiselana und Cervus elaphus. - Jahresschrift f. mitteldeut. Vorgeschichte des LfA Halle, 000-000, 98 Abb., 40 Taf.; (im Druck).

- (im Druck, b): Die Stellung von Dama (Cervidae, Mammalia) im System plesiometacarpaler Hirsche des Pleistozäns - Phylogenetische Rekonstruktion, Metrische Analyse. - Cour. Forsch.-Inst. Senckenberg, Frankfurt (im Druck).

Schaub, S (1941): Die kleine Hirschart aus dem Oberpliozän von Senéze (Haute-Loire). - Eclogae Geologicae Helveticae 36/2: 270-289.

ScHmid, E. (1965): Damhirsche im römischen Aust. Mitt. z. Ur- u. Frühgesch. d. Schweiz 29/4: 57-63, Abb. 55-60, 2 Tab.; Basel.

SEIfERT, M. (1990): Vegetationsgeschichtliche Entwicklung des Interglazials von Neumark-Nord. - EAZ 1: 10-16, Berlin.

Sutcliffe, A. (1960): Joint Mitnor Cave, Buckfastleigh - A Report on Excavations carried out during 1939 - 41 by the late A.H. Ogilvie. - Transactions of Torquay Natural History Society, 8/1: 3-28, 9 Taf; Torquay.

Manuskript eingegangen am: 6. November 1997 